\title{
Maximum-likelihood synchronization and channel estimation with multiuser detection in GFDMA
}

\author{
Hamidreza Shayanfar $^{1} \mid$ Hamid Saeedi-Sourck ${ }^{1}\left[\right.$ | Arman Farhang $^{2} \mid$ Linda E. Doyle $^{3}$
}

\author{
${ }^{1}$ Department of Electrical Engineering, \\ Yazd University, Yazd, Iran \\ ${ }^{2}$ School of Electrical and Electronic \\ Engineering, University College Dublin, \\ Dublin, Ireland \\ ${ }^{3}$ CONNECT, Trinity College Dublin, \\ Dublin, Ireland

\section{Correspondence} \\ Hamid Saeedi-Sourck, Department of \\ Electrical Engineering, Yazd University, \\ Yazd 89195-741, Iran. \\ Email: saeedi@yazd.ac.ir

\section{Funding information} \\ Science Foundation Ireland (SFI); \\ European Regional Development Fund, \\ Grant/Award Number: 13/RC/2077
}

\begin{abstract}
Accurate estimation and correction of channel distortions and carrier frequency offset (CFO) are of a great importance in any multicarrier communication system. Hence, in this paper, we propose data-aided CFO and channel estimation techniques for both multiuser uplink and downlink of the generalized frequency division multiple access (GFDMA). Our proposed solutions jointly estimate the $\mathrm{CFO}$ and channel responses based on the maximum-likelihood criterion. To simplify the implementation of the proposed estimation algorithms, we suggest a preamble composed of two similar Zadoff-Chu training sequences in a generalized frequency division multiplexing block. It is worth mentioning that our proposed technique can estimate both integer and fractional CFO values without any limitation on the acquisition range of CFO. In the uplink phase, each user aligns its carrier frequency with the base station using the estimated $\mathrm{CFO}$ in the downlink. However, the CFO estimates may get outdated for the uplink transmission. Thus, residual CFOs may still remain in the received signal at the base station. While being trivial in the downlink, CFO correction is a challenging task in the uplink. Thus, we also propose a joint $\mathrm{CFO}$ correction and channel equalization technique for the uplink of GFDMA systems. Finally, we evaluate our proposed estimation and correction algorithms in terms of estimation mean square error and bit error rate performance through simulations.
\end{abstract}

\section{1 | INTRODUCTION}

The demands of the emerging applications in the fifth generation $(5 \mathrm{G})$ of wireless communication systems such as machine-to-machine communications ${ }^{1}$ and the tactile Internet ${ }^{2}$ have necessitated the need for new signaling techniques addressing the shortcomings of orthogonal frequency division multiplexing (OFDM) while keeping its advantages. As a result, a number of waveforms have been reconsidered or new ones have emerged as candidates for inclusion in the physical layer of $5 \mathrm{G}$ networks. ${ }^{3-6 *}$

Generalized frequency division multiplexing (GFDM) is a potential $5 \mathrm{G}$ waveform that was first proposed by Fettweis et al in 2009. ${ }^{7}$ GFDM is a flexible multicarrier modulation scheme capable of spreading data symbols across the time-frequency grid. ${ }^{8}$ In GFDM, each subcarrier becomes localized in the frequency domain leading to more robustness

*Please ensure that you use the most up-to-date class file, available from the ETT Home Page at http://onlinelibrary.wiley.com/journal/10.1002/ (ISSN)2161-3915. 
against the carrier frequency offset (CFO) than OFDM. ${ }^{7}$ Localization of each subcarrier band relaxes the synchronization requirements in multiuser applications, especially in the uplink, compared with orthogonal frequency division multiple access (OFDMA) systems. ${ }^{3}$ Furthermore, GFDM deploys a single cyclic prefix (CP) per block of symbols rather than deployment of a CP per symbol, which is the case in OFDM. Hence, GFDM is more bandwidth efficient in time than OFDM.

One of the most challenging issues in multicarrier systems is their sensitivity to the CFO that is caused by the Doppler frequency shifts and local oscillator inaccuracies of user terminals. ${ }^{9} \mathrm{CFO}$, if not correctly estimated/compensated, misaligns the subcarriers in the frequency domain and leads to a large amount of interference. Frequency synchronization for the uplink is more difficult than that for the downlink, where each user's signal is determined by different frequency error. Then, base station (BS) has to estimate multiple CFOs. Furthermore, the second difficulty is the correction of the received signal using estimated CFOs. The sensitivity of GFDM to CFO was recently studied in the work of Aminjavaheri et al, ${ }^{10}$ where the authors consider the multiuser uplink scenario. Based on this study, GFDM suffers from a large amount of multiple access interference (MAI) due to the presence of multiple CFOs in the received signal at the BS. Therefore, accurate CFO estimation and correction play a pivotal role in the performance of both uplink and downlink communications. Additionally, accurate channel estimation is of a paramount importance in any practical communication system. To this end, Vilaipornsawai and $\mathrm{Jia}^{11}$ have proposed an estimation method that finds the channel impulse response (CIR) using a number of scattered pilots in a GFDM block through the least squares (LS) criterion. In a more recent work, ${ }^{12}$ Ehsanfar et al have proposed two channel estimation methods based on the LS and linear minimum mean square error (MSE) criteria that is applicable to multiple-input-multiple-output GFDM. In their methods, the CIR is estimated through utilizing scattered pilots among the data symbols in a GFDM block. However, in both works of Vilaipornsawai and Jia ${ }^{11}$ and Ehsanfar et al, ${ }^{12}$ perfect synchronization is considered, which is not the case in practice, and residual synchronization errors can adversely affect the channel estimation performance. In this paper, we focus on the CFO and channel estimation due to their impact on nonorthogonal waveforms.

There are two classes of CFO estimation techniques for GFDM in the literature, ie, blind and data aided. ${ }^{13-17}$ Kadur et al $^{13}$ proposed a blind CFO estimation method taking advantage of the nonorthogonality between GFDM subcarriers, ie, the leakage from the prototype filter sidelobes to the adjacent subcarriers. In the work of Wang and Lin, ${ }^{14}$ a CP-based blind maximum-likelihood (ML) CFO and timing offset (TO) synchronization technique was proposed. In the work of Gaspar et al, ${ }^{15}$ the OFDM-based well-known Schmidl and Cox synchronization technique ${ }^{18}$ is adopted to GFDM. This solution belongs to the class of data-aided estimators that is of interest to our paper. In a more recent work, ${ }^{16} \mathrm{TO}$ and CFO are estimated by deployment of the Schmidl and Cox algorithm, ${ }^{18}$ using an embedded training sequence in the middle of the payload. In this paper, the authors show the superiority of the CFO estimation accuracy using an isolated preamble proposed in the work of Gaspar et $\mathrm{al}^{15}$ to the deployment of an embedded training sequence placed in the middle of the payload. Matthé et $\mathrm{al}^{17}$ have studied the impact of CFO and TO in the uplink of the generalized frequency division multiple access (GFDMA) ${ }^{\dagger}$ and compared it with that of OFDM. In this study, the authors proposed a CFO and TO estimation algorithm based on the LS criterion using pilots scattered across a long GFDMA block. Additionally, they propose time domain and frequency domain methods for the correction of multiple CFOs and TOs.

The current literature on GFDM deals with the CFO and channel estimation separately while deploying different training sequences. As mentioned earlier, the residual CFO can significantly deteriorate the channel estimates. Thus, to tackle this problem, joint CFO and channel estimation methods need to be sought. To the best of our knowledge, there is no study available in the literature addressing joint CFO and channel estimation for GFDM. Hence, in this paper, we propose joint CFO and channel estimation algorithms for both downlink and uplink of GFDMA based on the ML criterion. Our proposed estimation techniques fall under the class of data-aided estimation methods as we find the CFO and channel estimates by deploying a known GFDM block as a preamble. To shorten the training overhead, we use only two GFDM symbols in the preamble block. We propose using two repetitions of a constant amplitude zero autocorrelation or Zadoff-Chu (ZC) sequence ${ }^{19}$ in the preamble. The advantage of this preamble is that it simplifies the estimation procedure and does not increase peak-to-average power ratio due to the constant amplitude. Additionally, our algorithm does not have any limitation on the CFO range in the downlink as it can estimate the integer and the fractional CFO values. Synchronization policy in practical systems is to precompensate the CFO effect in each user terminal before the uplink transmission. However, estimated CFO values in the downlink may be outdated for the uplink transmission. Consequently, residual CFOs may still remain in the received signal at the BS. ${ }^{9}$ It is worthy to note that we have derived

${ }^{\dagger}$ GFDMA is a combination of GFDM with frequency division multiple access where different users are multiplexed in different portions of the frequency band. 
the Cramer-Rau bound (CRB) for both uplink and downlink transmission to characterize the accuracy of our estimation techniques. The next step after CFO and channel estimation is the correction of their effects. While CFO correction and channel equalization are trivial tasks in the downlink, they are challenging in the uplink. ${ }^{9}$ Therefore, another contribution of this paper is to propose a joint frequency domain CFO correction and channel equalization technique for the uplink of GFDMA. Additionally, we evaluate the estimation MSE and bit error rate (BER) performance of our proposed techniques through simulations. Finally, we compare the MSE and BER performance of our proposed CFO estimation technique with the one in the work of Gaspar et $\mathrm{al}^{15}$ as it is the most relevant method to our solution due to the fact that they are both preamble-based estimators. It is worth mentioning that our proposed CFO and channel estimation techniques are not comparable with the other solutions in the literature, ${ }^{11-14,17}$ as they all belong to the class of blind or LS-based estimators.

The rest of this paper is organized as follows. Section 2 formulates the downlink and uplink system model for GFDMA. In Section 3, we present our proposed joint CFO and channel estimation techniques for both downlink and uplink scenarios. Section 4 presents downlink detection as well as our proposed joint CFO correction and channel equalization technique for the uplink detection. In Section 5, we numerically evaluate the performance of our proposed estimation and correction techniques. Finally, we conclude this paper in Section 6.

Notations. Matrices, vectors, and scalar quantities are denoted by boldface uppercase, boldface lowercase, and normal letters, respectively. The superscripts $(.)^{\mathbf{H}},(.)^{\mathbf{T}}$, and $(.)^{-1}$ denote Hermitian, transpose, and inverse of a matrix, respectively. $\mathbf{I}_{n}$ is the identity matrix of size $n \times n, \mathbf{0}_{n \times m}$ is the zero matrix of the size $n \times m$, and $\mathbf{1}_{n \times m}$ is the all-ones matrix of the size $n \times m$. $\mathbf{D}=\operatorname{diag}(\mathbf{a})$ is a diagonal matrix whose diagonal elements include the elements of the vector $\mathbf{a}$ and circshift(a, $m)$ denotes the downward circular shift operation with $m$ positions on the vector a. Finally, $\mathcal{F}_{M N}$ is the $M N$-point normalized discrete Fourier transform (DFT) matrix.

\section{2 | SYSTEM MODEL}

To pave the way for the derivations presented in this rest of the paper, in this section, we present the downlink and uplink system models.

\section{1 | Downlink transmission}

Consider the downlink of a GFDMA network with the total number of $U$ single antenna mobile terminals (MTs) that are communicating with a single antenna BS. Let $M$ be the total number of GFDMA symbols in a GFDMA block, $N=K U$ be the total number of subcarriers, and $K$ be the number of subcarriers allocated to each user. It is worth mentioning that, in this paper, we consider the block subcarrier allocation scheme (B-CAS) where a group of $K$ contiguous subcarriers is allocated to each user. The set of $K$ subcarriers assigned to a given user $u$ is denoted by $\Psi_{u}$, where $\bigcup_{u=0}^{U-1} \Psi_{u}=\{0,1, \ldots, N-$ $1\}$, and $\Psi_{i} \cap \Psi_{j}=\varnothing, \forall i \neq j$. Assuming that the $K \times M$ matrix $\mathbf{D}_{u}=\left[\mathbf{d}_{u}[0], \ldots, \mathbf{d}_{u}[M-1]\right]$ contains $M$ consecutive QAM data symbols belonging to user $u$ on its columns, the downlink transmit symbols of all the users can be rearranged in an $N \times M$ matrix $\mathbf{D}=\left[\mathbf{D}_{0}^{\mathrm{T}}, \ldots, \mathbf{D}_{U-1}^{\mathrm{T}}\right]^{\mathrm{T}}$. Therefore, the columns of $\mathbf{D}$ include the data symbols of all the users to be transmitted by the BS in a GFDMA block, ie,

$$
\mathbf{D}=[\mathbf{d}[0], \ldots, \mathbf{d}[M-1]]
$$

where $\mathbf{d}[m]=\left[d_{0, m}, \ldots, d_{N-1, m}\right]^{\mathrm{T}}$, with the entries $d_{n, m}$ corresponding to the data symbol to be transmitted on the $n$th subcarrier and the $m$ th time slot. In this paper, we consider the frequency spreading GFDM transmitter structure proposed in the work of Farhang-Boroujeny and Moradi. ${ }^{3}$ Thus, the GFDMA transmit block can be obtained as

$$
\mathbf{x}=\sum_{m=0}^{M-1} \operatorname{circshift}\left(\mathbf{x}_{m}, m \mathrm{~N}\right),
$$

where

$$
\mathbf{x}_{m}=\mathcal{F}_{M N}^{\mathrm{H}} \mathbf{C} \mathbf{d}_{e}[m]
$$


is the GFDMA transmit symbol at the time slot $m$, the $M N \times M N$ matrix $\mathbf{C}=\operatorname{circ}\{\mathbf{c}\}$ is circulant with the first column $\mathbf{c}=\left[\begin{array}{lllll}c_{0} & c_{1} \cdots c_{M-1} & 0 \cdots 0 & c_{M-1} \cdots c_{1}\end{array}\right]^{T}$, which contains the $2 M-1$ nonzero frequency domain coefficients of the prototype filter, and finally, the $M N \times 1$ vector

$$
\mathbf{d}_{\mathrm{e}}[m]=\left[\begin{array}{lllllll}
d_{0, m} & \mathbf{0}_{(M-1) \times 1} & d_{1, m} & \mathbf{0}_{(M-1) \times 1} & \cdots & d_{N-1, m} & \mathbf{0}_{(M-1) \times 1}
\end{array}\right]^{\mathrm{T}}
$$

is the $M$-fold expanded version of the column vector $\mathbf{d}[m]$.

Since, circular shift of a discrete time signal in the time domain is equivalent to the phase rotation in the frequency domain, we can rearrange (2) as

$$
\mathbf{x}=\sum_{m=0}^{M-1} \mathcal{F}_{M N}^{\mathrm{H}} \operatorname{diag}\left(\mathbf{a}_{m}\right) \mathcal{F}_{M N} \mathbf{x}_{m}
$$

where $\mathbf{a}_{m}=\left[1, e^{-\frac{j 2 \pi m}{M}}, \ldots, e^{-\frac{j 2 \pi m}{M}(M N-1)}\right]^{T}$. Hence, by substituting (3) in (5), we have

$$
\mathbf{x}=\mathcal{F}_{M N}^{\mathrm{H}}\left(\mathbf{C d}_{\mathrm{e}}[0]+\cdots+\operatorname{diag}\left(\mathbf{a}_{M-1}\right) \mathbf{C} \mathbf{d}_{\mathrm{e}}[M-1]\right) .
$$

As shown in Section 3, Equation (6) simplifies the CFO and channel estimation procedure. After constructing the GFDMA signal, $\mathbf{x}$, and appending the $\mathrm{CP}$, it is transmitted through the channel.

The $M N \times 1$ received signal vector $\mathbf{r}_{u}$ at the $u$ th MT after CP removal and in the presence of CFO and multipath channel can be shown as

$$
\mathbf{r}_{u}=\mathbf{E}_{u} \mathbf{X} \mathbf{h}_{u}+v_{u}
$$

where $v_{u} \sim \mathcal{C N}\left(0, \sigma_{v}^{2} \mathbf{I}_{M N}\right)$ is the complex additive white Gaussian noise vector with the variance $\sigma_{v}^{2}$. The vector $\mathbf{h}_{u}=$ $\left[h_{u, 0}, \ldots, h_{u, L-1}\right]^{\mathrm{T}}$ is the CIR between the BS and the $u$ th MT with the length $L$, and the $M N \times L$ matrix $\mathbf{X}$ contains the first $L$ columns of a circulant matrix whose first column is equal to $\mathbf{X}$. Multiplication of $\mathbf{X}$ to $\mathbf{h}_{u}$ is equivalent to the circular convolution of the GFDMA transmit signal $\mathbf{x}$ with the CIR $\mathbf{h}_{u}$. Finally, $\mathbf{E}_{u}=\operatorname{diag}\left(\boldsymbol{\varphi}_{u}\right)$ is the $M N \times M N$ CFO matrix where $\boldsymbol{\varphi}_{u}=\left[1, e^{\frac{j 2 \pi \varepsilon_{u}}{N}}, \ldots, e^{\frac{j 2 \pi \varepsilon_{u}(M N-1)}{N}}\right]^{\mathrm{T}}$ and $\varepsilon_{u}$ is the normalized CFO to the subcarrier spacing. It is worthy to note that the phase factor introduced by the CFO due to the CP samples is absorbed to the CIR.

\section{2 | Uplink transmission}

In the uplink transmission, the MTs are communicating with the BS through $U$ statistically independent multipath wireless channels. The same as in downlink transmission, in this paper, we consider the B-CAS for the uplink. Due to nonorthogonality and overlapping of adjacent subcarriers in GFDMA, the users need to use one subcarrier as a guard band on the edges of their bands to avoid multiuser interference. The same as in the downlink, subcarriers of distinct users should be mapped onto mutually exclusive subsets of the available subcarriers, ie, one subcarrier can be only used by one user.

The first step in the uplink transmission is subcarrier allocation. Therefore, the data symbols of user $u$ after subcarrier mapping can be shown as an $N \times M$ matrix

$$
\widetilde{\mathbf{D}}_{u}=\Gamma_{u} \mathbf{D}_{u},
$$

where $\Gamma_{u}$ is an $N \times K$ matrix whose columns include $K$ columns of an $N \times N$ identity matrix with the indices belonging to the subcarrier set of the $u$ th MT, ie, $\Psi_{u}$. Similar to (2), the transmit signal of a given user $u$ can be constructed as

$$
\mathbf{x}_{u}=\sum_{m=0}^{M-1} \operatorname{circshift}\left(\mathbf{x}_{m}^{u}, m N\right),
$$

where $\mathbf{x}_{m}^{u}=\mathcal{F}_{M N}^{\mathrm{H}} \mathbf{C} \tilde{\mathbf{d}}_{\mathrm{e}}^{u}[m]$ and $\tilde{\mathbf{d}}_{\mathrm{e}}^{u}[m]$ is $M$-fold expanded version of the $m$ th column of the matrix $\widetilde{\mathbf{D}}_{u}$, ie, the transmitted QAM symbols in the time slot $m$ from user $u$. After forming the GFDMA block $\mathbf{x}_{u}$ at the MT $u$, a CP is appended to the beginning of the GFDMA block. It is worth mentioning that the CP needs to be longer than both the maximum channel delay spread and the two-way propagation delay to avoid self- and multiuser interference due to the channel delay spread and the TOs of the users, respectively. ${ }^{9}$ Hence, the users are quasi-synchronous in time and the phase factors due to different users' delays are absorbed to their corresponding channel responses.

After discarding the $\mathrm{CP}$ from the received signal at the $\mathrm{BS}$ and with the assumption of having perfect power control for all the users, we have

$$
\mathbf{r}=\sum_{u=0}^{U-1} \mathbf{E}_{u} \mathbf{X}_{u} \mathbf{h}_{u}+v,
$$




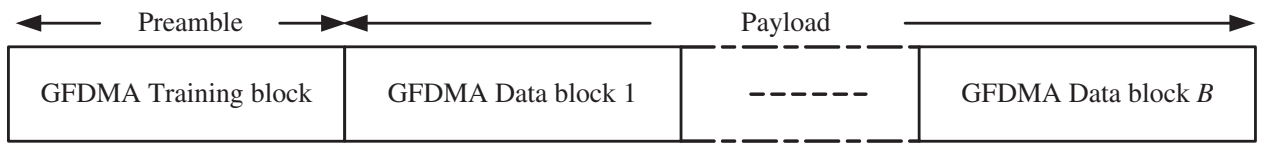

FIGURE 1 Generalized frequency division multiplexing downlink/uplink data packet structure. GFDMA, generalized frequency division multiple access

where $\mathbf{E}_{u}$ and $\mathbf{h}_{u}$ have the same definitions as in Section 2.1, $\mathbf{X}_{u}$ is an $M N \times L$ matrix containing the first $L$ columns of a circulant matrix with the first column equal to the vector $\mathbf{x}_{u}$, and $v$ is the additive white Gaussian noise vector with the variance of $\sigma_{v}^{2}$. From (10), one may realize that multiplication of $\mathbf{X}_{u}$ to the CIR vector $\mathbf{h}_{u}$ realizes the circular convolution of the $u$ th MT signal with its corresponding CIR.

\section{3 | PROPOSED JOINT CFO AND CHANNEL ESTIMATION}

In this section, we propose joint CFO and channel estimation algorithms for both downlink and uplink transmissions. Our proposed estimation methods that are presented in the following sections utilize a known GFDM training block that is transmitted in the beginning of the GFDMA data packet, see Figure 1. It is worthy to note that the channel is assumed to be static during transmission of the whole data packet.

\section{1 | Downlink CFO and channel estimation}

Our proposed joint CFO and channel estimation method works based on the minimum variance unbiased estimator principles. ${ }^{20}$ According to (7), the CIR coefficients of the user $u$ in the downlink can be obtained as

$$
\hat{\mathbf{h}}_{u}=\left(\mathbf{X}^{\mathrm{H}} \mathbf{X}\right)^{-1} \mathbf{X}^{\mathrm{H}} \mathbf{E}_{u}^{\mathrm{H}} \mathbf{r}_{u} .
$$

Substituting (11) into (7), we have

$$
\mathbf{r}_{u}=\mathbf{E}_{u} \mathbf{X}\left(\mathbf{X}^{\mathrm{H}} \mathbf{X}\right)^{-1} \mathbf{X}^{\mathrm{H}} \mathbf{E}_{u}^{\mathrm{H}} \mathbf{r}_{u}+v_{u} .
$$

Thus, the CFO of the user $u$ can be estimated through the ML criterion. To this end, the CFO estimation is performed through grid search as

$$
\hat{\varepsilon}_{u}=\underset{\varepsilon}{\operatorname{argmax}}\left\{\ln \mathrm{P}\left(\mathbf{r}_{u} \mid \varepsilon\right)\right\},
$$

where $\ln \mathrm{P}\left(\mathbf{r}_{u} \mid \varepsilon\right)$ is the natural logarithm of the conditional probability density function of $\mathbf{r}_{u}$ given $\varepsilon$. Ignoring the irrelevant terms to $\varepsilon$, we have

$$
\begin{aligned}
\hat{\varepsilon}_{u}=\underset{\varepsilon}{\operatorname{argmin}}\left\{\left(\mathbf{r}_{u}-\mathbf{E X}\left(\mathbf{X}^{\mathrm{H}} \mathbf{X}\right)^{-1} \mathbf{X}^{\mathrm{H}} \mathbf{E}^{\mathrm{H}} \mathbf{r}_{u}\right)^{\mathrm{H}}\right. \\
\\
\left.\times\left(\mathbf{r}_{u}-\mathbf{E X}\left(\mathbf{X}^{\mathrm{H}} \mathbf{X}\right)^{-1} \mathbf{X}^{\mathrm{H}} \mathbf{E}^{\mathrm{H}} \mathbf{r}_{u}\right)\right\},
\end{aligned}
$$

where $\mathbf{E}$ is obtained in the same way as $\mathbf{E}_{u}$ by substitution of $\varepsilon$ rather than $\varepsilon_{u}$. After some mathematical manipulations, (14) can be simplified to

$$
\hat{\varepsilon}_{u}=\underset{\varepsilon}{\operatorname{argmax}}\left\{\mathbf{r}_{u}^{\mathrm{H}} \mathbf{E X}\left(\mathbf{X}^{\mathrm{H}} \mathbf{X}\right)^{-1} \mathbf{X}^{\mathrm{H}} \mathbf{E}^{\mathrm{H}} \mathbf{r}_{u}\right\} .
$$

In the packet format that is shown in Figure 1, $M=2$ is considered for the training block, ie, the preamble, to reduce the overhead it imposes to the system, and according to our transceiver filters, odd values of $M$ are considered for the payload. Furthermore, as shown in the work of Nimr et al, ${ }^{21}$ with proper filter design, even values of $M$ can be used for the payload. To lower the CFO estimation complexity, we propose to utilize the same ZC training sequence ${ }^{19}$ on both symbols in the preamble to make the matrix $\mathbf{X}^{\mathrm{H}} \mathbf{X}$ in (15) diagonal. The $N \times 1 \mathrm{ZC}$ sequence vector is defined as $\psi=$ $\frac{1}{\sqrt{N}}\left[1, e^{\frac{j \beta \pi}{N}}, \ldots, e^{\frac{j \beta \pi}{N}(N-1)^{2}}\right]^{\mathrm{T}}$, where $\beta$ is an integer parameter relatively prime with respect to $N$ and $N$ is the length of the sequence. It is known that cyclically shifted versions of the ZC sequence $\psi$ constitute a set of orthogonal basis vectors. Hence, using (6), it can be shown that the orthogonal property of the ZC sequence is preserved after GFDM modulation. Hence, we have

$$
\mathbf{X}^{\mathrm{H}} \mathbf{X}=\rho \mathbf{I}_{L}
$$


where $\rho=2 c_{0}^{2}$ (see Appendix A). Then, (15) is simplified to

$$
\begin{aligned}
\hat{\varepsilon}_{u} & =\underset{\varepsilon}{\operatorname{argmax}}\left\{\mathbf{r}_{u}^{\mathrm{H}} \mathbf{E} \mathbf{X} \mathbf{X}^{\mathrm{H}} \mathbf{E}^{\mathrm{H}} \mathbf{r}_{u}\right\} \\
& =\underset{\varepsilon}{\operatorname{argmax}}\left\{\left\|\mathbf{r}_{u}^{\mathrm{H}} \mathbf{E X}\right\|_{2}\right\} .
\end{aligned}
$$

Finally, through substitution of (17) into (11), the channel estimate of the user $u$ can be obtained as

$$
\hat{\mathbf{h}}_{u}=(1 / \rho) \mathbf{X}^{\mathrm{H}} \hat{\mathbf{E}}_{u}^{\mathrm{H}} \mathbf{r}_{u},
$$

where $\hat{\mathbf{E}}_{u}=\operatorname{diag}\left\{\hat{\varphi}_{u}\right\}$ and $\hat{\varphi}_{u}=\left[1, \ldots, e^{\frac{j 2 \pi(M N-1)}{N}} \hat{\varepsilon}_{u}\right]^{\mathrm{T}}$.

\section{2 | Proposed search algorithm}

The CFO estimation method proposed in the work of Gaspar et al ${ }^{15}$ has an acquisition CFO range limited to only half-subcarrier spacing. However, our proposed method in (17) does not have any limitation in terms of the CFO range. In particular, we estimate the integer and the fractional part of $\mathrm{CFO}$ within the range $[-N / 2,+N / 2)$. CFO estimation using (17) can be performed through the conventional search with small and fixed search steps. The down side of this approach is its high computational complexity, especially for large CFO values. To address the complexity issue, in our method, we take advantage of the particular properties of the cost function $\mathcal{G}=\left\|\mathbf{r}_{u}^{\mathrm{H}} \mathbf{E X}\right\|_{2}$ when the ZC sequence is utilized. In this case, search is only performed at the neighborhood of $\beta$ parts of the cost function. ${ }^{\ddagger}$ Assuming the high signal-to-noise (SNR) scenario, the received signal $\mathbf{r}_{u}$ in (17) is approximately equal to $\mathbf{r}_{u} \approx \mathbf{E}_{u} \mathbf{X h}$. By substitution of $\mathbf{r}_{u}$ in (17), we have

$$
\hat{\varepsilon}_{u}=\underset{\varepsilon}{\operatorname{argmax}}\left\{\left\|\mathbf{h}_{u}^{\mathrm{H}} \mathbf{X}^{\mathrm{H}} \Phi_{u} \mathbf{X}\right\|_{2}\right\},
$$

where

$$
\begin{aligned}
\Phi_{u} & =\mathbf{E}_{u}^{\mathrm{H}} \mathbf{E} \\
& =\operatorname{diag}\left(\left[\begin{array}{ll}
\xi_{u}^{\mathrm{T}} & \lambda \xi_{u}^{\mathrm{T}}
\end{array}\right]^{\mathrm{T}}\right),
\end{aligned}
$$

and $\xi_{u}=\left[1, e^{\frac{j 2 \pi \Delta f_{u}}{N}}, \ldots, e^{\frac{j 2 \pi \Delta f_{u}(N-1)}{N}}\right]^{\mathrm{T}}$. In addition, $\Delta f_{u}=\varepsilon-\varepsilon_{u}$ and $\lambda=e^{j 2 \pi \Delta f_{u}}$. For CFO estimation, we can exploit the properties of ZC sequence. Concentrating on the term $\mathbf{X}^{\mathrm{H}} \Phi_{u} \mathbf{X}$ in (19) and defining an $L \times L$ matrix $\mathbf{Q}_{u}$ as

$$
\mathbf{Q}_{u}=\mathbf{X}^{\mathrm{H}} \Phi_{u} \mathbf{X}
$$

it can be shown that the elements $\left[\mathbf{Q}_{u}\right]_{l_{1}, l_{2}}$ can be obtained as

$$
\left[\mathbf{Q}_{u}\right]_{l_{1}, l_{2}}=(1+\lambda) \mathbf{p}_{l_{1}}^{\mathrm{H}} \operatorname{diag}\left\{\xi_{u}\right\} \mathbf{p}_{l_{2}}, \quad l_{1}, l_{2}=0, \ldots, L-1,
$$

where $\mathbf{p}_{l}=\operatorname{circshift}\left(\frac{2}{\sqrt{2}} c_{0}\left(\mathcal{F}_{N}^{\mathrm{H}} \boldsymbol{\psi}\right), l\right)$. Expanding (22), we have

$$
\begin{aligned}
{\left[\mathbf{Q}_{u}\right]_{l_{1}, l_{2}} } & =(1+\lambda) \sum_{n=0}^{N-1}\left[p_{l_{1}}^{*}[n] p_{l_{2}}[n]\right] e^{\frac{-j 2 \pi \Delta f_{u} n}{N}} \\
& =2 c_{0}^{2}(1+\lambda) \sum_{n=0}^{N-1}\left[\sum_{k_{1}=0}^{N-1} e^{\frac{-j \beta \pi k_{1}^{2}}{N}} e^{\frac{j 2 \pi k_{1} l_{1}}{N}} e^{\frac{-j 2 \pi k_{1} n}{N}} \sum_{k_{2}=0}^{N-1} e^{\frac{j \beta \pi k_{2}^{2}}{N}} e^{\frac{-j 2 \pi k_{2} l_{2}}{N}} e^{\frac{j 2 \pi k_{2} n}{N}}\right] e^{\frac{-j 2 \pi \Delta f_{u} n}{N}} \\
& =2 c_{0}^{2}(1+\lambda) \sum_{k_{1}=0}^{N-1} \sum_{k_{2}=0}^{N-1} e^{\frac{j \beta \pi\left(k_{2}^{2}-k_{1}^{2}\right)}{N}} e^{\frac{j 2 \pi\left(l_{1} k_{1}-l_{2} k_{2}\right)}{N}}\left[\sum_{n=0}^{N-1} e^{\frac{-j 2 \pi\left(k_{1}-k_{2}+\Delta f_{u}\right) n}{N}}\right]
\end{aligned}
$$

In (23), $\Delta f_{u}=\Delta f_{u, \mathrm{i}}+\Delta f_{u, \mathrm{f}}$, where $\Delta f_{u, \mathrm{i}}$ and $\Delta f_{u, \mathrm{f}}$ are the integer and fractional parts of $\Delta f_{u}$, respectively. With regard to $-N / 2 \leq \Delta f_{u, \mathrm{i}} \leq N / 2-1$ and assuming $\Delta f_{u, \mathrm{f}}=0$, it can be shown that

$$
\begin{aligned}
\sum_{n=0}^{N-1} e^{\frac{-j 2 \pi\left(k_{1}-k_{2}+\Delta f_{u}\right) n}{N}} & =\sum_{n=0}^{N-1} e^{\frac{-j 2 \pi\left(k_{1}-k_{2}+\Delta f_{u, \mathrm{i}}\right) n}{N}} \\
& = \begin{cases}N, & k_{1}-k_{2}+\Delta f_{u, \mathrm{i}}=0 ; \\
0, & \text { O.W. }\end{cases}
\end{aligned}
$$

\footnotetext{
${ }^{\ddagger}$ As it will be shown in the following, for simplicity of computing, $\beta$ is usually chosen to be 3 .
} 


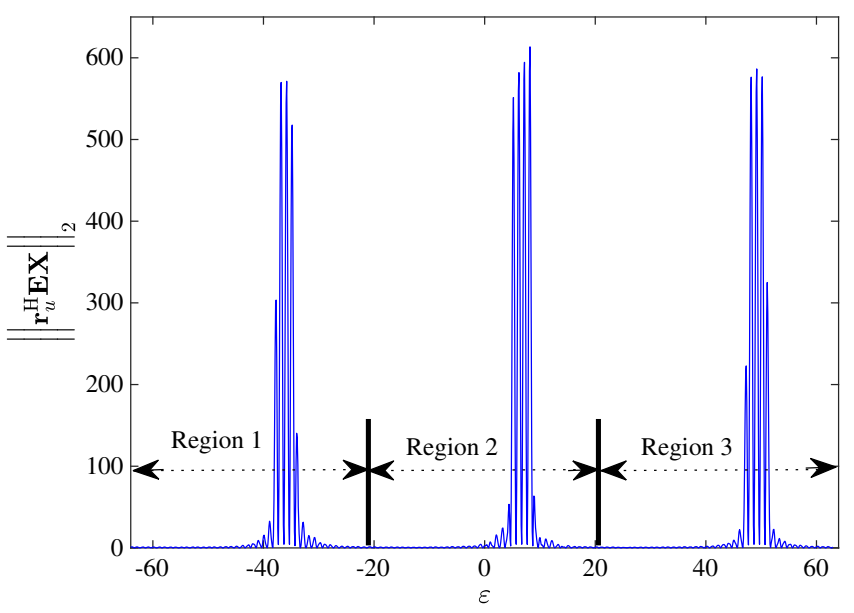

FIGURE 2 Typical cost function for (19) with $\varepsilon_{u}=8.1716, N=128$, and $\beta=3$

Therefore, (23) is nonzero only when $\Delta f_{u, \mathrm{i}}=k_{2}-k_{1}$. Consequently, (23) can be simplified to

$$
\begin{aligned}
{\left[\mathbf{Q}_{u}\right]_{l_{1}, l_{2}} } & =2 N c_{0}^{2}(1+\lambda) \sum_{k_{1}=0}^{N-1} e^{\frac{j \beta \pi\left(\Delta f_{u, \mathrm{i}}\right)\left(2 k_{1}+\Delta f_{u, \mathrm{i}}\right)}{N}} e^{\frac{j 2 \pi\left(l_{1} k_{1}-l_{2} \Delta f_{u, \mathrm{i}}-l_{2} k_{1}\right)}{N}} \\
& =2 N c_{0}^{2}(1+\lambda) e^{\frac{j \beta \pi \Delta f_{u, \mathrm{i}}^{2}}{N}} e^{\frac{-j 2 \pi l_{2} \Delta f_{u, \mathrm{i}}}{N}} \sum_{k_{1}=0}^{N-1} e^{\frac{j 2 \pi k_{1}\left(\beta \Delta f_{u, \mathrm{i}}+l_{1}-l_{2}\right)}{N}}
\end{aligned}
$$

Following the same lines of derivation as in (23), we can obtain nonzero elements presented in (25) only for $\beta \Delta f_{u, \mathrm{i}}+l_{1}-l_{2}=$ $i N$. Given $-N / 2 \leq \Delta f_{u, \mathrm{i}} \leq N / 2-1$ and assuming that $N \gg L$, we can conclude

$$
-\left\lfloor\frac{\beta}{2}\right\rfloor \leq i \leq\left\lfloor\frac{\beta}{2}\right\rfloor
$$

Inequality (26) reveals that the parts of the cost function with high values depend on parameter $\beta$, as shown in Figure 2. Thus, we propose a computationally efficient search algorithm reducing the search region into the neighborhood of only $\beta$ parts of the cost function. In the presence of fractional CFO values, $\left|\Delta f_{u, f}\right| \leq 0.5$, similar results to the ones presented above can be achieved. Our proposed search algorithm is summarized in Algorithm 1.

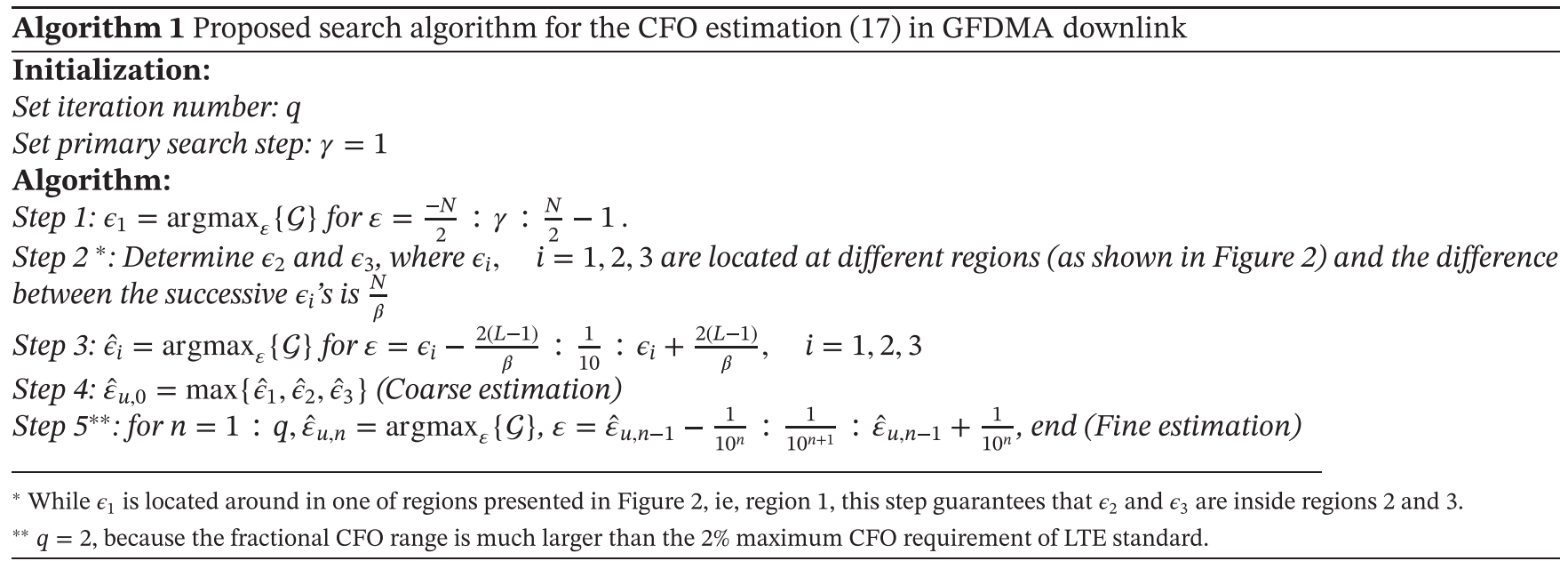




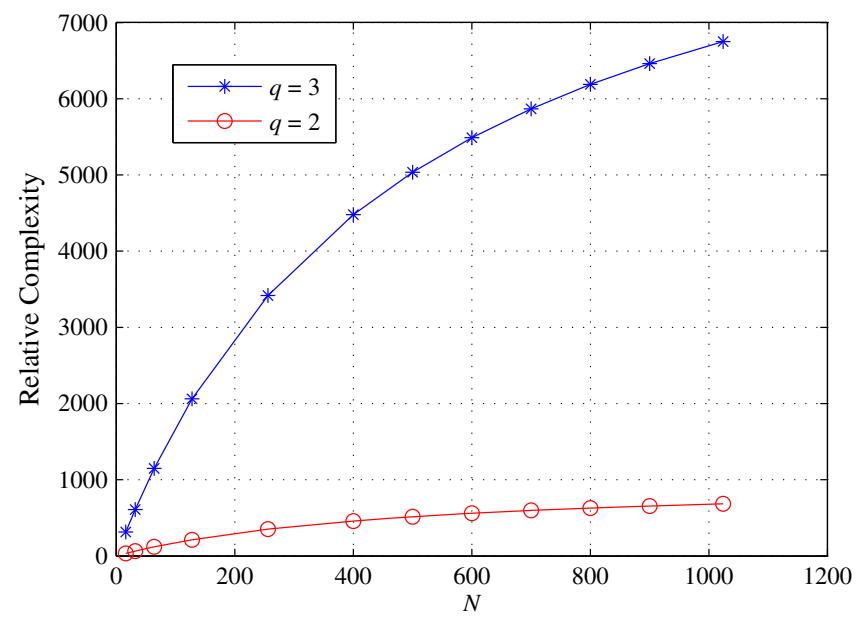

FIGURE 3 Relative computational complexity of the conventional search compared with the proposed search algorithm, where $\beta=3$ and $L=11$

\subsection{1 | Complexity analysis}

As mentioned before, the proposed ML CFO estimation in (17) with conventional search method results in a high computational complexity. Assuming the computational complexity for calculation of the cost function $\{\mathcal{G}\}$ equal to $\mathcal{C M}$, the computational complexity of the conventional search with the step size $10^{-(q+1)}$ is $\left(N \times 10^{(q+1)} \times C \mathcal{M}\right)$. In comparison, the computational complexity of our proposed search algorithm with $\gamma=1$ is reduced to $\left\{N+\left(3 \times\left(\frac{4(L-1)}{\beta}+1\right) \times 10\right)+((2 \times 10+\right.$ 1) $\times q)\} \times C \mathcal{M}$. In other words, for a large number of subcarriers, the computational complexity of our proposed search algorithm is reduced by about $q$ orders of magnitude compared with the conventional search method. Figure 3 presents the relative complexity of the conventional search algorithm compared with our proposed method. As the figure shows, our method leads to two to three orders of magnitude complexity reduction.

\section{3 | Uplink CFO and channel estimation}

In uplink, different users are multiplexed in the frequency domain and their received signals at the BS experience different CFOs and wireless channels. Different from the downlink, our proposed CFO and channel estimation algorithm in the uplink transmission is performed in the frequency domain. This is due to the fact that different users are multiplexed in the frequency domain.

After taking $M N$-point DFT from (10), the received signal in the frequency domain is obtained as

$$
\mathbf{y}=\sum_{u=0}^{U-1} \mathcal{F}_{M N} \mathbf{E}_{u} \mathbf{X}_{u} \mathbf{h}_{u}+\mathcal{F}_{M N} \nu
$$

By separation of the band belonging to a given user $u$ through $\mathbf{y}_{u}=\mathbf{P}_{u} \mathbf{y}$, we can estimate the CFO and CIR of the user $u$ similar to the downlink as

$$
\hat{\mathbf{h}}_{u}=\left(\mathbf{X}_{u}^{\mathrm{H}} \mathbf{X}_{u}\right)^{-1} \mathbf{X}_{u}^{\mathrm{H}} \mathbf{E}_{u}^{\mathrm{H}} \mathcal{F}_{M N}^{\mathrm{H}} \mathbf{y}_{u}
$$

and

$$
\hat{\varepsilon}_{u}=\underset{\varepsilon_{u}}{\operatorname{argmax}}\left\{\mathbf{y}_{u}^{\mathrm{H}} \mathcal{F}_{M N} \mathbf{E}_{u} \mathbf{X}_{u}\left(\mathbf{X}_{u}^{\mathrm{H}} \mathbf{X}_{u}\right)^{-1} \mathbf{X}_{u}^{\mathrm{H}} \mathbf{E}_{u}^{\mathrm{H}} \mathcal{F}_{M N}^{\mathrm{H}} \mathbf{y}_{u}\right\},
$$

where $\mathbf{P}_{u}=\operatorname{diag}\left\{\left[\mathbf{0}_{1 \times u M K}, \mathbf{1}_{1 \times M K}, \mathbf{0}_{1 \times(M N-(u+1) M K)}\right]^{\mathrm{T}}\right\}$ is an $M N \times M N$ diagonal matrix that separates the signal portion belonging to user $u$ in the frequency domain. As we will show in Section 4, channel frequency response estimation is more accurate than the CIR estimation of (28). Thus, to estimate the channel frequency response, we first rearrange (27) as

$$
\mathbf{y}=\Lambda \mathbf{z}+\mathcal{F}_{M N} v,
$$

where

$$
\mathbf{z}=\mathcal{H}_{\mathrm{f}} \mathbf{x}_{\mathrm{f}},
$$


$\mathbf{x}_{\mathrm{f}}=\mathcal{F}_{M N} \sum_{u=0}^{U-1} \mathbf{x}_{u}$, and $\mathcal{H}_{\mathrm{f}}$ is a diagonal compound channel matrix whose diagonal elements include the frequency domain channel responses corresponding to different active users in different parts of the band. The matrix

$$
\Lambda=\sum_{u=0}^{U-1} \mathcal{F}_{M N} \mathbf{E}_{u} \mathcal{F}_{M N}^{\mathrm{H}} \mathbf{P}_{u}
$$

is called the interference matrix due to the CFOs of all the users. It is worth mentioning that since GFDM is based on transmission of $M N$ tones similar to OFDM,$^{3}$ the interference matrix $\Lambda$ has a similar structure to its counterparts in OFDMA uplink systems presented in the work of Morelli et al. ${ }^{9}$ Finally, $\mathbf{P}_{u}$ chooses the columns of $\mathcal{F}_{M N} \mathbf{E}_{u} \mathcal{F}_{M N}^{\mathrm{H}}$ that are associated with the sample indices of the user $u$ in the frequency domain.

In order to estimate the elements of $\mathcal{H}_{\mathrm{f}}$, the effect of all the users' CFOs on the training block needs to be corrected first. To this end, we deploy the frequency domain correction of multiple CFOs based on the minimum MSE (MMSE) criterion. Using the estimated CFO values of all the users through (29), we have

$$
\hat{\mathbf{z}}_{\mathrm{MMSE}}=\left(\hat{\Lambda}^{\mathrm{H}} \hat{\Lambda}+\sigma_{\nu}^{2} \mathbf{I}_{N}\right)^{-1} \hat{\Lambda}^{\mathrm{H}} \mathbf{y},
$$

where $\hat{\Lambda}$ can be obtained by inserting $\hat{\mathbf{E}}_{u}$ into (32). Finally, recalling (31), the compound channel can be obtained through

$$
\hat{\mathcal{H}}_{\mathrm{f}}=\left(\operatorname{diag}\left(\mathbf{x}_{\mathrm{f}}\right)\right)^{-1} \operatorname{diag}\left(\hat{\mathbf{z}}_{\mathrm{MMSE}}\right) .
$$

It is worth mentioning that CRBs for the proposed CFO and channel estimation techniques in Sections 3.1 and 3.3 have been derived in Appendices B.1 and B.2, respectively. We have used the derived CRBs as benchmarks for performance evaluation of our proposed estimation methods.

\section{4 | CFO CORRECTION AND CHANNEL EQUALIZATION}

After finding the frequency misalignments of the users and their channel estimates, the next step is to correct the effect of the CFO and equalize the multipath channel. In the following sections, we present the data detection procedure in the downlink and uplink scenarios. It is worth noting that, in the rest of this paper, we represent the number of GFDMA symbols in the training and the data blocks as $M_{\mathrm{t}}$ and $M_{\mathrm{d}}$, respectively. This is due to the fact that the preamble and the data blocks are comprised of a different number of symbols. Even though $M_{\mathrm{t}}$ can be set equal to $M_{\mathrm{d}}$, we consider $M_{\mathrm{t}}=2$ to minimize the training overhead.

\section{1 | Downlink detection}

As mentioned earlier, $\mathrm{CFO}$ correction in the downlink is a trivial task. This is due to the fact that there exists only a single CFO in the received downlink signal at a given MT $u$, ie, $\varepsilon_{u}$. Thus, after estimation of $\varepsilon_{u}$ through (17), its effect on the payload can be simply compensated by multiplication of the diagonal matrix $\hat{\mathbf{E}}_{u}^{\mathrm{H}}$ to the received signal at the MT $u$, ie, $\mathbf{r}_{u}$ in Equation (7) where $\mathbf{X}$ contains the payload data. Then, using the channel estimate obtained in (18), the resulting signal from $\hat{\mathbf{E}}_{u}^{\mathrm{H}} \mathbf{r}_{u}$ can be equalized and the data can be detected through the frequency domain equalizer and the receiver structure discussed in the work of Farhang et al, ${ }^{22}$ respectively. Finally, each user can extract its corresponding time-frequency data symbols from the output of its GFDMA receiver.

\section{2 | Uplink detection}

Correction of the frequency misalignments in the uplink is more challenging than downlink. This is because there exist multiple CFOs in the received uplink signal at the BS leading to a great amount of MAI if not compensated. To this end, in this section, we propose a method to jointly correct the effects of the multiple CFOs and the users' multipath channels.

Recalling (30) and (31), the compound frequency domain signal vector $\mathbf{x}_{\mathbf{f}}$ can be estimated by solving a linear system of equations as

$$
\hat{\mathbf{x}}_{\mathrm{f}, \mathrm{MMSE}}=\left(\left(\hat{\Lambda} \hat{\mathcal{H}}_{\mathrm{f}, \text { int. }}\right)^{\mathrm{H}} \hat{\Lambda} \hat{\mathcal{H}}_{\mathrm{f}, \text { int. }}+\sigma_{v}^{2} \mathbf{I}_{M N}\right)^{-1}\left(\hat{\Lambda} \hat{\mathcal{H}}_{\mathrm{f}, \text { int. }}\right)^{\mathrm{H}} \mathbf{y}
$$


TABLE 1 Simulation parameters for the generalized frequency division multiple access (GFDMA) system

\begin{tabular}{ccl} 
Parameter & Value & \multicolumn{1}{c}{ Description } \\
\hline$N$ & 128 & Number of subcarriers \\
$M_{\mathrm{t}}$ & 2 & Number of symbols in the training block \\
$M_{\mathrm{d}}$ & 3 & Number of symbols in the payload data block \\
$B$ & 5 & Number of GFDMA data blocks \\
$U$ & 8 & Number of users \\
$K$ & 16 & Number of alocated contiguous subcarriers to each user \\
$N_{\mathrm{g}}$ & 1 & Number of guard subcarriers in uplink \\
$N_{\mathrm{CP}}$ & $\lfloor 0.125 N\rfloor$ & Length of cyclic prefix \\
$\alpha$ & 0.1 & Roll-off factor (raised cosine filter) \\
$\mu$ & $16-\mathrm{QAM}$ & Modulation type \\
$\beta$ & 3 (downlink), 7 (uplink) & Zadoff-Chu training sequence parameter \\
\hline
\end{tabular}

where $\hat{\mathcal{H}}_{\mathrm{f} \text {,int. }}$ is a diagonal matrix of the size $M_{\mathrm{d}} N \times M_{\mathrm{d}} N$ that includes the interpolated version of the channel frequency response that is estimated in (34) with the interpolation factor of $M_{\mathrm{d}} / 2$. This is due to the fact that $M_{\mathrm{t}}=2$ for the training block while $M_{\mathrm{d}} \neq M_{\mathrm{t}}$.

Since, the effects of the multiple CFOs and the users' multipath channels are jointly compensated in (35), for a low complexity implementation of the BS receiver, the resulting time domain vector from $\mathcal{F}_{N M_{\mathrm{d}}}^{\mathrm{H}} \hat{\mathbf{x}}_{\mathrm{f}, \mathrm{MMSE}}$ can be straightforwardly fed into the zero-forcing receiver structure proposed in the work of Farhang et al. ${ }^{22}$ Let the $N \times M$ compound data matrix $\hat{\mathbf{D}}=[\hat{\mathbf{d}}[0], \ldots, \hat{\mathbf{d}}[M-1]]$, ie, the output of the GFDMA receiver, include the detected time-frequency data symbols of all the users and $\hat{\mathbf{d}}[m]=\left[\hat{d}_{0, m}, \ldots, \hat{d}_{N-1, m}\right]^{\mathrm{T}}$, the estimated data symbols of the $u$ th MT can be extracted from $\hat{\mathbf{D}}$ in a $K \times M$ matrix as $\hat{\mathbf{D}}_{u}=\Gamma_{u}^{\mathrm{T}} \hat{\mathbf{D}}$.

\section{5 | SIMULATION RESULTS}

In this section, we present our simulation results for the proposed techniques in Sections 3 and 4 for both downlink and uplink scenarios. The simulation parameters are presented in Table 1. As shown in this table, we consider $U=8$ users and allocate a block of $K=16$ contiguous subcarriers to each user. For the uplink scenario, one guard subcarrier is considered on the edges of the users' bands to ensure orthogonality among the users. In our simulations, we use the extended typical urban channel model, ie, an LTE channel model. ${ }^{23}$ The subcarrier spacing is set to be the same as in the LTE standard, ie, $15 \mathrm{kHz}$. The CP length is $\lfloor 0.125 \mathrm{~N}\rfloor$, ie, long enough to accommodate the wireless channel delay spread as well as two-way propagation delay. We use a raised cosine prototype filter with the roll-off factor of $\alpha=0.1$ and ZC training sequence with different $\beta=3$ and $\beta=7$ for downlink and uplink transmission, respectively. ${ }^{24}$ The CFO values in our simulations are chosen randomly and independently from the uniform distribution.

Figure 4 compares the MSE performance of our proposed CFO estimation technique using our low complexity search algorithm, presented in Section 3.2, versus the conventional search. As shown in this figure, the MSE performance of our proposed search algorithm follows the conventional approach for the SNRs larger than $10 \mathrm{~dB}$. Thus, to improve the estimation accuracy in SNRs lower than $10 \mathrm{~dB}$, we recommend the conventional search at the expense of an increased computational complexity.

In Figure 5, we investigate the MSE performance of our proposed joint CFO and channel estimation techniques in the downlink transmission, where the CFO values are chosen in the interval $[-N / 2, N / 2)$. As shown in this figure, the MSE performance of our proposed estimators improve as SNR increases and our results are close to the relevant CRBs derived in Appendix B.1. In addition, as mentioned earlier, for the proposed CFO estimation in SNRs lower than $10 \mathrm{~dB}$, the MSE values of the conventional search are used to improve the accuracy of estimation. In Figure 6, we compare the MSE performance of our proposed CFO estimation technique with that of the one proposed in the work of Gaspar et al..$^{15}$ As this Figure shows, the MSE of our proposed technique coincides with the CRB while being superior to the MSE of the method proposed in the aforementioned work. ${ }^{15}$ As mentioned earlier, the proposed method in the aforementioned work ${ }^{15}$ is limited to the $\mathrm{CFO}$ values within the interval $\varepsilon_{u} \in[-1 / 2,1 / 2)$ while our proposed estimator does not have any limitation on the CFO range. Thus, for a fair comparison, in this Figure, we choose the CFOs within the range $\varepsilon_{u} \in[-1 / 2,1 / 2)$. 


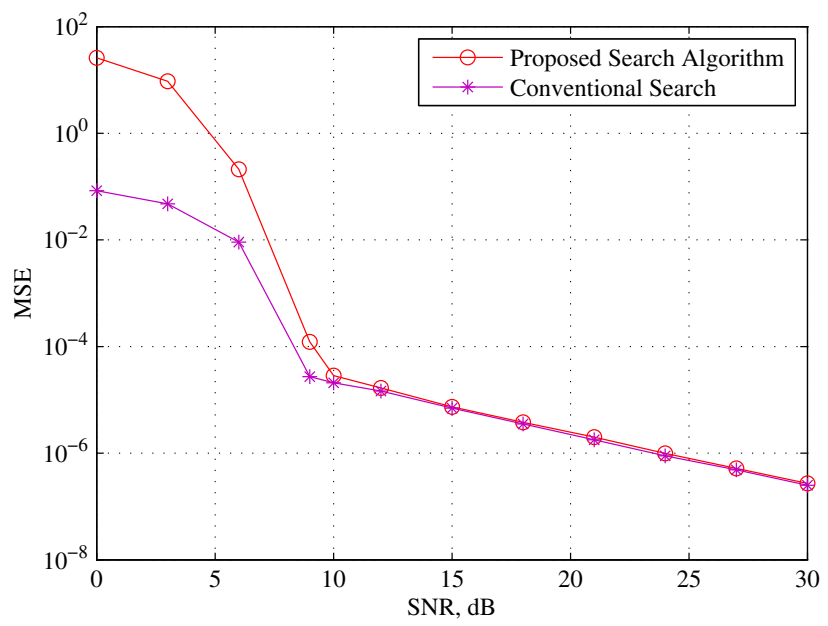

FIGURE 4 Mean square error (MSE) comparison between the conventional search approach and the proposed search algorithm. SNR, signal-to-noise ratio

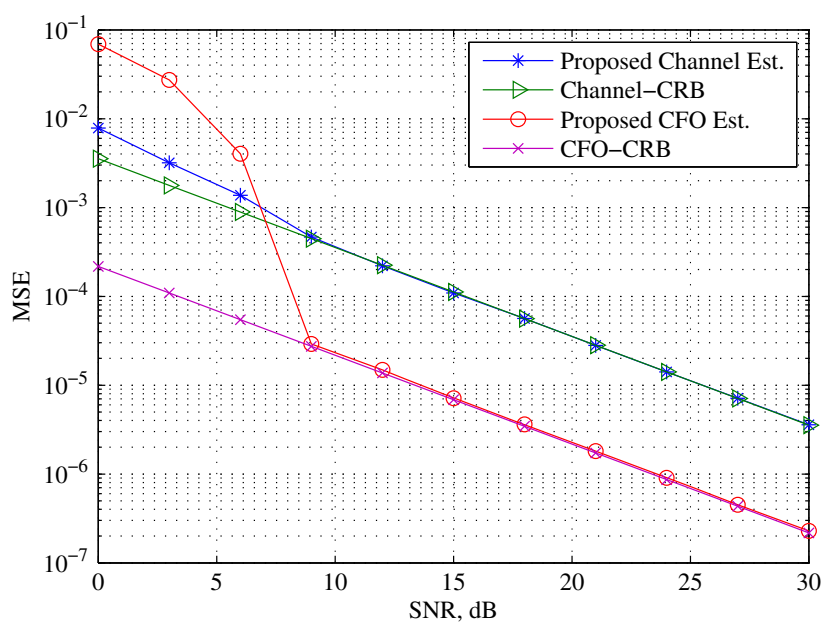

FIGURE 5 Mean square error (MSE) performance of the proposed joint carrier frequency offset (CFO) and channel estimation for the downlink. CRB, Cramer-Rau bound

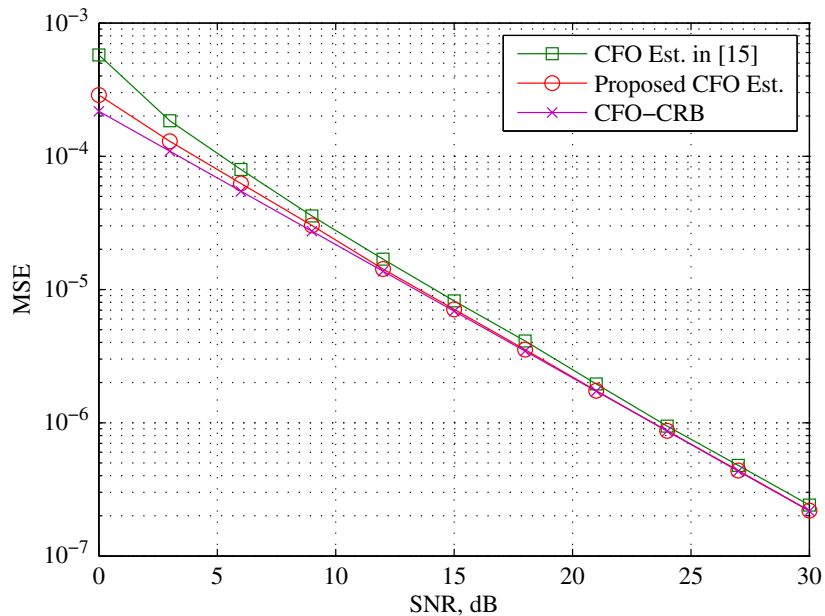

FIGURE 6 The mean square error (MSE) performance comparison of the proposed estimator versus the one proposed in the work of Gaspar et al, ${ }^{15}$ where $\varepsilon_{u} \in[-1 / 2,1 / 2)$. CFO, carrier frequency offset; CRB, Cramer-Rau bound; SNR, signal-to-noise ratio 


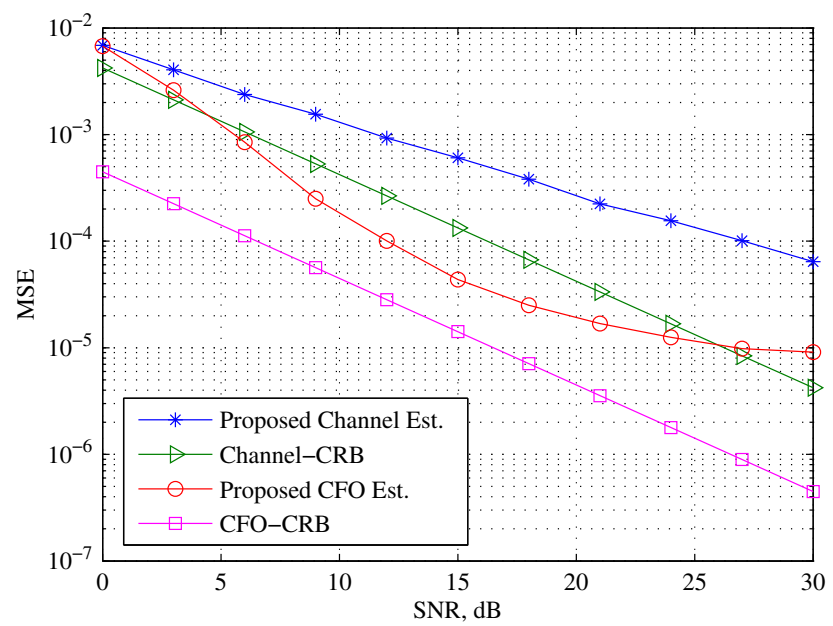

FIGURE 7 Mean square error (MSE) of the joint carrier frequency offset (CFO) and channel estimation for the uplink. CRB, Cramer-Rau bound; SNR, signal-to-noise ratio

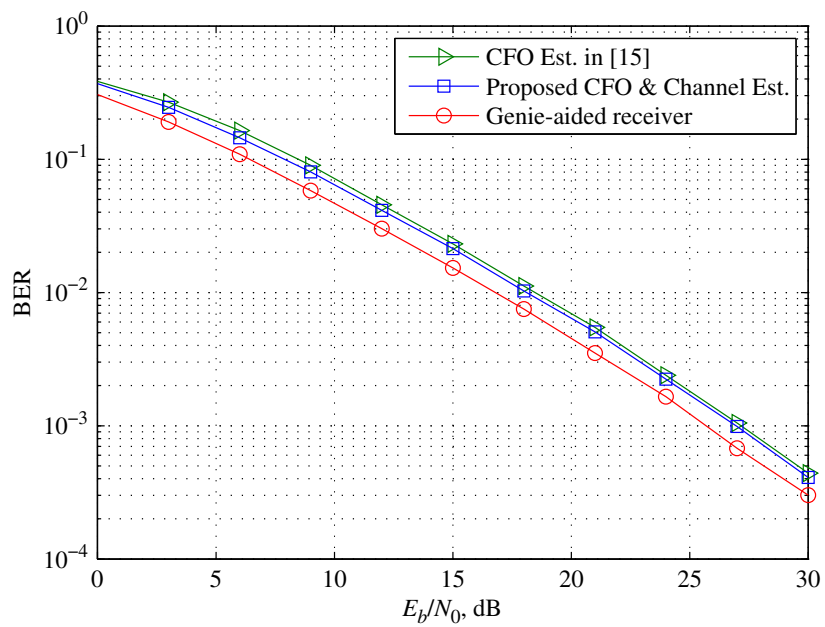

FIGURE 8 Downlink bit error rate (BER) performance. CFO, carrier frequency offset

In addition, by precise comparison between Figures 5 and 6 and according to different CFO ranges for both figures, the performance of the proposed estimator is independent of the CFO range for high SNR values.

Figure 7 shows the average MSE performance of our proposed joint CFO and channel estimation technique for the uplink. CFO values are chosen in the interval $\frac{-0.5}{M_{\mathrm{t}}}<\varepsilon_{u}<\frac{0.5}{M_{\mathrm{t}}}$ similar to the Matthé et al. ${ }^{17}$ This figure shows the performance of the proposed estimator in comparison with the derived CRB in Appendix B.2 as a benchmark. As shown in this figure, an error floor of around $10^{-5}$ is observed for the CFO estimates. This is due to the presence of the MAI in the frequency domain signal portion of a given user $u$, ie, $\mathbf{y}_{u}$ in (29).

Finally, Figures 8 and 9 show the BER performance of the proposed schemes in the presence of multipath channel and CFO for both downlink and uplink. In the BER results, 16-QAM modulation scheme with convolution coding and the code rate of $1 / 2$ is considered. We have provided the BER performance when the perfect knowledge of the CFOs and the channel responses are available at the receiver known as the genie-aided receiver. This curve is used as a benchmark for comparison with our proposed techniques in Sections 3 and 4. As shown in Figure 8, the downlink BER performance of our proposed estimation technique is close to the BER of the case with perfect knowledge of the channel and CFO. This shows the efficacy of our proposed joint CFO and channel estimation technique for the downlink. Figure 9 evaluates the performance of our proposed joint CFO and channel estimation and compensation in the uplink. As shown in this figure, the proposed CFO correction and channel equalization remains solid and its BER performance follows the reference curve, ie, when all the users are fully synchronized and their channel responses are perfectly known at the BS. However, 


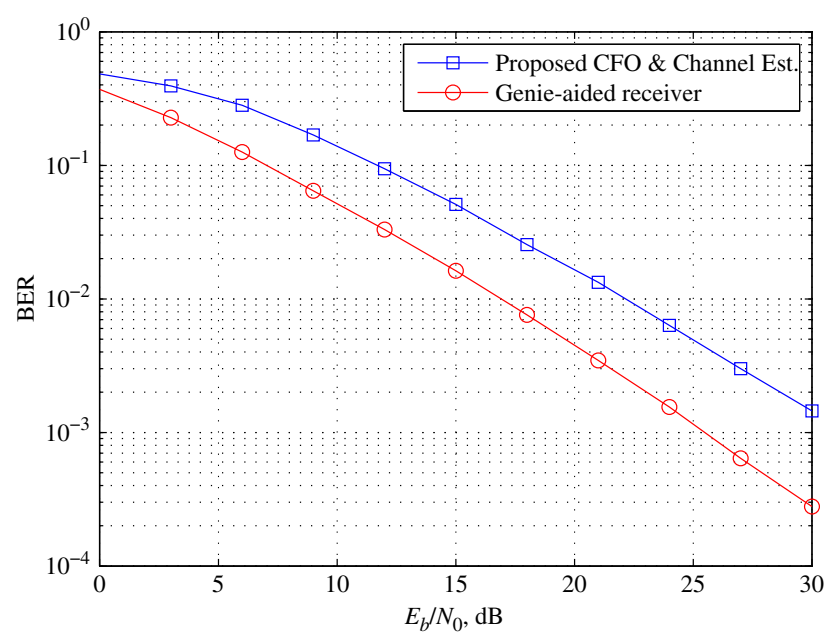

FIGURE 9 Uplink bit error rate (BER) performance. CFO, carrier frequency offset

our proposed joint $\mathrm{CFO}$ correction and channel equalization with the estimated channel and CFO values suffer from a BER performance penalty of around $5 \mathrm{~dB}$. This is due to the fact that the $\mathrm{CFO}$ and channel estimates suffer from inaccuracy due to the presence of residual MAI.

\section{6 | CONCLUSION}

In this paper, we have proposed joint CFO and channel estimation algorithms for GFDMA systems in both uplink and downlink. Our proposed solutions are based on the ML criterion. We have suggested utilization of two similar ZC sequences in a GFDM block as a preamble, which simplifies implementation of our proposed estimation techniques. To ease the CFO estimation further in the downlink, we have proposed a low complexity search algorithm. Additionally, our proposed CFO estimation method does not have any limitation on the CFO range. Apart from CFO estimation in the uplink, we have also proposed a joint multiple CFO correction and channel equalization technique. Finally, we have assessed the performance of our proposed estimation and correction techniques in both downlink and uplink through simulations in terms of the MSE of the estimation and BER performance, respectively.

In this paper, we proposed a simple search method to reduce the amount of computations through a step-size adjustment procedure. This work can be pursued in different directions in the future, namely, (1) further simplification of the search procedure through more advanced techniques such as genetic or branch and bound algorithms and (2) finding a closed-form CFO expression that does not involve any search procedure.

\section{ACKNOWLEDGEMENTS}

This publication has emanated from research conducted with the financial support of Science Foundation Ireland (SFI) and is co-funded under the European Regional Development Fund under Grant Number 13/RC/2077.

\section{ORCID}

Hamid Saeedi-Sourck (Dttp://orcid.org/0000-0002-8363-5030

\section{REFERENCES}

1. Chen M, Wan J, Li F. Machine-to-machine communications: architectures, standards and applications. KSII Trans Internet Inf Syst. 2012;6(2):480-497.

2. Fettweis GP. The tactile internet: applications and challenges. IEEE Veh Technol Mag. 2014;9(1):64-70.

3. Farhang-Boroujeny B, Moradi H. OFDM inspired waveforms for 5G. IEEE Commun Surv Tutor. 2016;18(4):2474-2492.

4. Farhang A, Marchetti N, Figueiredo F, Miranda JP. Massive MIMO and waveform design for 5th generation wireless communication systems. Paper presented at: 1st International Conference on 5G for Ubiquitous Connectivity; November 26-28, 2014; Akaslompolo, Finland. 
5. Wunder G, Jung P, Kasparick M, et al. 5GNOW: non-orthogonal, asynchronous waveforms for future mobile applications. IEEE Commun Mag. 2014;52(2):97-105.

6. RezazadehReyhani A, Farhang A, Farhang-Boroujeny B. Circularly pulse-shaped waveforms for 5G: options and comparisons. Paper presented at: 2015 IEEE Global Communications Conference (GLOBECOM); December 6-10, 2015; San Diego, CA.

7. Fettweis G, Krondorf M, Bittner S. GFDM - generalized frequency division multiplexing. Paper presented at: VTC Spring 2009 - IEEE 69 th Vehicular Technology Conference; April 26-29, 2009; Barcelona, Spain.

8. Michailow N, Datta R, Krone S, Lentmaier M, Fettweis G. Generalized frequency division multiplexing: a flexible multi-carrier modulation scheme for 5th generation cellular networks. In: Proceedings of the German Microwave Conference 2012 (GeMiC'12); March 12-14, 2012; Ilmenau, Germany.

9. Morelli M, Kuo C-CJ, Pun M-O. Synchronization techniques for orthogonal frequency division multiple access (OFDMA): a tutorial review. Proc IEEE. 2007;95(7):1394-1427.

10. Aminjavaheri A, Farhang A, RezazadehReyhani A, Farhang-Boroujeny B. Impact of timing and frequency offsets on multicarrier waveform candidates for 5G. Paper presented at: 2015 IEEE Signal Processing and Signal Processing Education Workshop (SP/SPE); August 9-12, 2015; Salt Lake City, UT.

11. Vilaipornsawai U, Jia M. Scattered-pilot channel estimation for GFDM. Paper presented at: 2014 IEEE Wireless Communications and Networking Conference (WCNC); April 6-9, 2014; Istanbul, Turkey.

12. Ehsanfar S, Matthe M, Zhang D, Fettweis G. A study of pilot-aided channel estimation in MIMO-GFDM systems. Paper presented at: WSA 2016. 20th International ITG Workshop on Smart Antennas; March 9-11, 2016; Munich, Germany.

13. Kadur T, Gaspar I, Michailow N, Fettweis G. Non-data aided frequency synchronization exploiting ICI in non-orthogonal systems. Paper presented at: 2014 IEEE 80th Vehicular Technology Conference (VTC2014-Fall); September 14-17, 2014; Vancouver, Canada.

14. Wang P-S, Lin DW. Maximum-likelihood blind synchronization for GFDM systems. IEEE Sig Process Lett. 2016;23(6):790-794.

15. Gaspar IS, Mendes LL, Michailow N, Fettweis G. A synchronization technique for generalized frequency division multiplexing. EURASIP J Adv Sig Process. 2014:(1)67.

16. Gaspar IS, Fettweis G. An embedded midamble synchronization approach for generalized frequency division multiplexing. Paper presented at: 2015 IEEE Global Communications Conference (GLOBECOM); December 6-10, 2015; San Diego, CA.

17. Matthé M, Mendes LL, Fettweis G. Asynchronous multi-user uplink transmission with generalized frequency division multiplexing. Paper presented at: 2015 IEEE International Conference on Communication Workshop (ICCW); June 8-12, 2015; London, UK.

18. Schmidl TM, Cox DC. Robust frequency and timing synchronization for OFDM. IEEE Trans Commun. 1997;45(12):1613-1621.

19. Geetha Priya C, Suganthi M. Combined PAPR reduction and frequency offset estimation using precoded Zadoff-Chu OFDM in WLAN system. Paper presented at: 2010 3rd International Conference on Computer Science and Information Technology; July 9-11, 2010; Chengdu, China.

20. Kay SM. Fundamentals of Statistical Signal Processing, Volume I: Estimation Theory. Upper Saddle River, NJ: Prentice-Hall, Inc.; 1993.

21. Nimr A, Matthé M, Zhang D, Fettweis G. Optimal Radix-2 FFT compatible filters for GFDM. IEEE Commun Lett. 2017;21(7):1497-1500.

22. Farhang A, Marchetti N, Doyle LE. Low-complexity modem design for GFDM. IEEE Trans Signal Process. 2016;64(6):1507-1518.

23. Yang WB, Souryal M. LTE Physical Layer Performance Analysis. National Institute of Standards and Technology, United States Department of Commerce; Gaithersburg, MD: 2014.

24. Matthé M, Michailow N, Gaspar I, Fettweis G. Influence of pulse shaping on bit error rate performance and out of band radiation of Generalized Frequency Division Multiplexing. Paper presented at: 2014 IEEE International Conference on Communications Workshops (ICC); June 10-14, 2014; Sydney, Australia.

25. Besson O, Stoica P. On parameter estimation of MIMO flat-fading channels with frequency offsets. IEEE Trans Signal Process. 2003;51(3):602-613.

26. Pun M-O, Morelli M, Kuo C-CJ. Maximum-likelihood synchronization and channel estimation for OFDMA uplink transmissions. IEEE Trans Commun. 2006;54(4):726-736.

How to cite this article: Shayanfar H, Saeedi-Sourck H, Farhang A, Doyle LE. Maximum-likelihood synchronization and channel estimation with multiuser detection in GFDMA. Trans Emerging Tel Tech. 2018;29:e3424. https://doi.org/10.1002/ett.3424

\section{APPENDIX A : DERIVATION OF $\mathrm{X}^{\mathrm{H}} \mathrm{X}$ FOR THE PROPOSED PREAMBLE}

Recalling (6),

$$
\mathbf{x}=\mathcal{F}_{M N}^{H}\left(\mathbf{C d}_{e}[0]+\cdots+\operatorname{diag}\left(\mathbf{a}_{M-1}\right) \mathbf{C d} \mathbf{d}_{e}[M-1]\right) .
$$

We set $M=2$ and $\mathbf{d}_{\mathrm{e}}[0]=\mathbf{d}_{\mathrm{e}}[1]=\boldsymbol{\psi}_{\mathrm{e}}$, where $\boldsymbol{\psi}_{\mathrm{e}}$ is the twofold expanded version of the $N \times 1$ vector $\boldsymbol{\psi}$. Hence, (A1) can be written as

$$
\mathbf{x}=\mathcal{F}_{2 N}^{\mathrm{H}}\left(\mathbf{C} \boldsymbol{\psi}_{\mathrm{e}}+\operatorname{diag}\left(\mathbf{a}_{1}\right) \mathbf{C} \boldsymbol{\psi}_{\mathrm{e}}\right)
$$


By left factorization of the matrix $\mathbf{C} \boldsymbol{\psi}_{\mathrm{e}}$ and recalling the vector $\mathbf{a}_{1}=\left[1, e^{-j \pi}, \ldots, e^{-j \pi(2 N-1)}\right]^{\mathrm{T}}$, we have

$$
\begin{aligned}
\mathbf{x} & =\mathcal{F}_{2 N}^{\mathrm{H}}\left(\mathbf{I}_{2 N}+\operatorname{diag}\left(\mathbf{a}_{1}\right)\right) \mathbf{C} \boldsymbol{\psi}_{\mathrm{e}} \\
& =\mathcal{F}_{2 N}^{\mathrm{H}} \operatorname{diag}\left(\left[\begin{array}{lll}
2 & 0 \cdots 2 & 0
\end{array}\right]\right) \mathbf{C} \boldsymbol{\psi}_{\mathrm{e}} \\
& =2 c_{0} \mathcal{F}_{2 N}^{\mathrm{H}} \boldsymbol{\psi}_{\mathrm{e}}=\frac{2}{\sqrt{2}} c_{0}\left[\left(\mathcal{F}_{N}^{\mathrm{H}} \boldsymbol{\psi}\right)^{\mathrm{T}},\left(\mathcal{F}_{N}^{\mathrm{H}} \boldsymbol{\psi}\right)^{\mathrm{T}}\right]^{\mathrm{T}} .
\end{aligned}
$$

From (A3) and due to the fact that multiplication of the $N$-point inverse DFT matrix to $\psi$ preserves the orthogonality property of the ZC sequence, the parameter $\rho$ in (16) is obtained as

$$
\rho=\mathbf{x}^{\mathrm{H}} \mathbf{x}=2 c_{0}^{2} .
$$

\section{APPENDIX B : DERIVATION OF CRB}

In this section, the $\mathrm{CRB}$ of the joint $\mathrm{CFO}$ and channel estimation are derived for both downlink and uplink transmissions.

\section{B.1 | Downlink CRB}

Recalling (7) and ignoring the index $u$ for the sake of simplicity without loss of generality, we have

$$
\mathbf{r}=\mathbf{E X h}+v=\mathcal{P}+v,
$$

where $\mathcal{P} \triangleq \mathbf{E X h}$. Sorting the unknown parameters to be estimated in a vector $\boldsymbol{\eta}$, we have

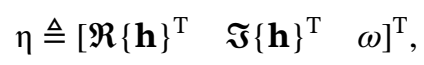

where $\mathfrak{R}\{\mathbf{h}\}$ and $\mathfrak{\Im}\{\mathbf{h}\}$ are the real and imaginary parts of $\mathbf{h}$, respectively, and $\omega \triangleq \frac{2 \pi \varepsilon}{N}$. The Fisher information matrix (FIM) for the estimation of $\boldsymbol{\eta}$ can be found using the Slepian-Bangs formula ${ }^{25}$

$$
\begin{aligned}
\mathbf{F} & =\frac{2}{\sigma_{v}^{2}} \mathfrak{R}\left\{\frac{\partial \mathcal{P}^{\mathrm{H}}}{\partial \boldsymbol{\eta}} \frac{\partial \mathcal{P}}{\partial \boldsymbol{\eta}^{\mathrm{T}}}\right\} \\
& =\frac{2}{\sigma_{v}^{2}} \mathfrak{R}\left\{\sum_{n=0}^{M N-1} \frac{\partial \mathcal{P}^{\mathrm{H}}(n)}{\partial \boldsymbol{\eta}} \frac{\partial \mathcal{P}(n)}{\partial \boldsymbol{\eta}^{\mathrm{T}}}\right\},
\end{aligned}
$$

where

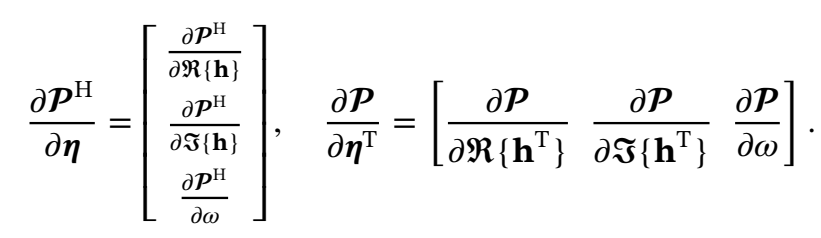

It can be shown that the elements in (B4) are qual to

$$
\begin{aligned}
& \frac{\partial \mathcal{P}}{\partial \mathfrak{R}\left\{\mathbf{h}^{\mathrm{T}}\right\}}=\mathbf{E X}, \\
& \frac{\partial \mathcal{P}}{\partial \mathfrak{I}\left\{\mathbf{h}^{\mathrm{T}}\right\}}=j \mathbf{E X}, \\
& \frac{\partial \mathcal{P}}{\partial \omega}=j \mathbf{G}_{n} \mathbf{E X h},
\end{aligned}
$$

where the diagonal matrix $\mathbf{G}_{n}=\operatorname{diag}\left\{[0,1, \ldots, M N-1]^{\mathrm{T}}\right\}$. Additionally $\frac{\partial \boldsymbol{p}^{\mathrm{H}}}{\partial \boldsymbol{\eta}}$ can be obtained in the same way from (B5)-(B7). Therefore, the FIM according to (B3) can be obtained as

$$
\mathbf{F}=\frac{2}{\sigma_{\nu}^{2}}\left[\begin{array}{ccc}
\mathfrak{R}\{\mathbf{B}\} & -\mathfrak{I}\{\mathbf{B}\} & -\mathfrak{I}\{A\} \\
\mathfrak{I}\{\mathbf{B}\} & \mathfrak{R}\{\mathbf{B}\} & \mathfrak{R}\{A\} \\
\mathfrak{I}\{A\}^{\mathrm{H}} & \mathfrak{R}\{A\}^{\mathrm{H}} & \mathfrak{R}\{q\}
\end{array}\right],
$$


where $\mathbf{B}=\mathbf{X}^{\mathrm{H}} \mathbf{E}^{\mathrm{H}} \mathbf{E X}, \boldsymbol{A}=\mathbf{X}^{\mathrm{H}} \mathbf{E}^{\mathrm{H}} \mathbf{D}_{n} \mathbf{E X h}$ and $q=\mathbf{h}^{\mathrm{H}} \mathbf{X}^{\mathrm{H}} \mathbf{E}^{\mathrm{H}} \mathbf{D}_{n}^{2} \mathbf{E X h}$. Finally, CRB is obtained as the inverse of the FIM ${ }^{25}$

$$
\operatorname{CRB}(\boldsymbol{\eta})=\mathbf{F}^{1} \text {. }
$$

Using matrix inversion lemma, ${ }^{25} \mathbf{F}$ can be inverted as

$$
\mathbf{C R B}(\boldsymbol{\eta})=\frac{\sigma_{\nu}^{2}}{2}\left[\begin{array}{ccc}
\mathfrak{R}\left\{\mathbf{B}^{-1}\right\} & -\mathfrak{I}\left\{\mathbf{B}^{-1}\right\} & 0 \\
\mathfrak{I}\left\{\mathbf{B}^{-1}\right\} & \mathfrak{R}\left\{\mathbf{B}^{-1}\right\} & 0 \\
0 & 0 & 0
\end{array}\right]+\Omega\left[\begin{array}{c}
\mathfrak{I}\left\{\mathbf{B}^{-1} A\right\} \\
-\mathfrak{R}\left\{\mathbf{B}^{-1} A\right\} \\
\mathrm{I}
\end{array}\right]\left[\mathfrak{I}_{\left\{\mathbf{B}^{-1} A\right\}^{\mathrm{T}}-\mathfrak{R}\left\{\mathbf{B}^{-1} A\right\}^{\mathrm{T}}} \mathbf{I}\right],
$$

where $\Omega=\left[\Re\left\{q-\mathbf{A}^{\mathrm{H}} \mathbf{B}^{-1} A\right\}\right]^{-1}$. Hence, the CRB associated with the frequency offset becomes

$$
\mathbf{C R B}(\varepsilon)=\frac{N^{2} \sigma_{v}^{2}}{8 \pi^{2}}\left[\Re\left\{q-\mathbf{A}^{H} \mathbf{B}^{-1} A\right\}\right]^{-} 1,
$$

and the CRB for the real and imaginary parts of the channel coefficients are given as

$$
\begin{aligned}
& \mathbf{C R B}[\Re\{\mathbf{h}\}]=\frac{\sigma_{v}^{2}}{2}\left[\Re\left\{\mathbf{B}^{-1}\right\}+\Omega \times \mathfrak{J}\left\{\mathbf{B}^{-1} A\right\} \times \mathfrak{J}\left\{\mathbf{B}^{-1} A\right\}^{\mathrm{T}}\right], \\
& \mathbf{C R B}[\mathfrak{\Im}\{\mathbf{h}\}]=\frac{\sigma_{v}^{2}}{2}\left[\Re\left\{\mathbf{B}^{-1}\right\}+\Omega \times \Re\left\{\mathbf{B}^{-1} A\right\} \times \Re\left\{\mathbf{B}^{-1} A\right\}^{\mathrm{T}}\right] .
\end{aligned}
$$

\section{B.2 | Uplink CRB}

According to (30), we have

$$
\mathbf{y}=\Lambda \boldsymbol{H}_{\mathrm{f}} \mathbf{x}_{\mathrm{f}}+\boldsymbol{F}_{M N} \nu
$$

To obtain CRB for CFO and channel estimation in the uplink, we rewrite (B14) as

$$
\mathbf{y}=\Lambda \mathcal{X}_{\mathrm{f}} \tilde{\mathbf{h}}_{\mathrm{f}}+\boldsymbol{F}_{M N} \boldsymbol{v}
$$

where $\mathcal{X}_{\mathrm{f}}=\operatorname{diag}\left(\mathbf{x}_{\mathrm{f}}\right)$ and $\tilde{\mathbf{h}}_{\mathrm{f}}$ is a compound channel vector whose elements include the frequency domain channel responses corresponding to different users active in different parts of the band. Therefore, we can define the vector of the unknown parameters to be estimated as

$$
\eta \triangleq\left[R\left\{\tilde{\mathbf{h}}_{\mathrm{f}}\right\}^{\mathrm{T}} \quad \mathfrak{\Im}\left\{\tilde{\mathbf{h}}_{\mathrm{f}}\right\}^{\mathrm{T}} \quad \omega\right]^{\mathrm{T}},
$$

where $\boldsymbol{\omega}=\left[\omega_{0}, \omega_{1}, \ldots, \omega_{U-1}\right]^{\mathrm{T}}$ and $\omega_{u}=\frac{2 \pi \varepsilon_{u}}{N}$. The components of the FIM can be calculated in the same way as in the downlink. Hence, through some mathematical manipulations, FIM can be obtained as

$$
\mathbf{F}=\frac{2}{\sigma_{v}^{2}}\left[\begin{array}{ccc}
\mathfrak{R}\left\{\mathbf{T}^{\mathrm{H}} \mathbf{T}\right\} & -\mathfrak{I}\left\{\mathbf{T}^{\mathrm{H}} \mathbf{T}\right\} & -\mathfrak{I}\left\{\mathbf{T}^{\mathrm{H}} \mathbf{Q}\right\} \\
\mathfrak{I}\left\{\mathbf{T}^{\mathrm{H}} \mathbf{T}\right\} & \mathfrak{R}\left\{\mathbf{T}^{\mathrm{H}} \mathbf{T}\right\} & \mathfrak{R}\left\{\mathbf{T}^{\mathrm{H}} \mathbf{Q}\right\} \\
\mathfrak{J}\left\{\mathbf{Q}^{\mathrm{H}} \mathbf{T}\right\} & \mathfrak{R}\left\{\mathbf{Q}^{\mathrm{H}} \mathbf{T}\right\} & \mathfrak{R}\left\{\mathbf{Q}^{\mathrm{H}} \mathbf{Q}\right\}
\end{array}\right]
$$

where $\mathbf{T}=\Lambda \mathcal{X}_{\mathrm{f}}, \mathbf{Q}=\left[\mathbf{q}_{0}, \mathbf{q}_{1}, \ldots, \mathbf{q}_{U-1}\right]$, and $\mathbf{q}_{u}=\boldsymbol{F}_{M N} \mathbf{D}_{n} \mathbf{E}_{u} \boldsymbol{F}_{M N}^{\mathrm{H}} \mathbf{P}_{u} \mathcal{X}_{\mathrm{f}} \tilde{\mathbf{h}}_{\mathrm{f}} \cdot{ }^{26}$ Thus, the CRB for the CFO estimates, $\varepsilon_{u}$, can be derived as

$$
\mathbf{C R B}\left(\varepsilon_{u}\right)=\frac{N^{2} \sigma_{v}^{2}}{8 \pi^{2}}\left[\Re\left\{\mathbf{Q}^{\mathrm{H}} \mathbf{Q}-\left(\mathbf{T}^{\mathrm{H}} \mathbf{Q}\right)^{\mathrm{H}}\left(\mathbf{T}^{\mathrm{H}} \mathbf{T}\right)^{-1} \mathbf{T}^{\mathrm{H}} \mathbf{Q}\right\}\right]_{u, u}^{-1} .
$$

Finally, the CRB for the real and imaginary parts of the channel estimates $\Re\left(\tilde{\mathbf{h}}_{\mathrm{f}}\right)$ and $\mathfrak{\Im}\left(\tilde{\mathbf{h}}^{\mathbf{f}}\right)$, respectively, can be obtained as

$$
\mathbf{C R B}\left[\Re\left\{\tilde{\mathbf{h}}_{\mathrm{f}}\right\}\right]=\frac{\sigma_{v}^{2}}{2}\left[\mathfrak{R}\left\{\left(\mathbf{T}^{\mathrm{H}} \mathbf{T}\right)^{-1}\right\}+\Im\left\{\left(\mathbf{T}^{\mathrm{H}} \mathbf{T}\right)^{-1} \mathbf{T}^{\mathrm{H}} \mathbf{Q}\right\} \times \Omega \times \mathfrak{I}\left\{\left(\mathbf{T}^{\mathrm{H}} \mathbf{T}\right)^{-1} \mathbf{T}^{\mathrm{H}} \mathbf{Q}\right\}^{\mathrm{T}}\right],
$$

and

$$
\left.\mathbf{C R B}\left[\Im\left\{\tilde{\mathbf{h}}_{\mathrm{f}}\right\}\right]=\frac{\sigma_{v}^{2}}{2}\left[\Re\left\{\left(\mathbf{T}^{\mathrm{H}} \mathbf{T}\right)^{-1}\right\}+\mathfrak{R}\left\{\left(\mathbf{T}^{\mathrm{H}} \mathbf{T}\right)^{-1} \mathbf{T}^{\mathrm{H}} \mathbf{Q}\right\}\right] \times \Omega \times \mathfrak{R}\left\{\left(\mathbf{T}^{\mathrm{H}} \mathbf{T}\right)^{-1} \mathbf{T}^{\mathrm{H}} \mathbf{Q}\right\}^{\mathrm{T}}\right]
$$

where $\Omega=\left[\Re\left\{\mathbf{Q}^{\mathrm{H}} \mathbf{Q}-\left(\mathbf{T}^{\mathrm{H}} \mathbf{Q}\right)^{\mathrm{H}}\left(\mathbf{T}^{\mathrm{H}} \mathbf{T}\right)^{-1} \mathbf{T}^{\mathrm{H}} \mathbf{Q}\right\}\right]^{-1}$. 